\title{
Taste Profile Characterization of Chinese Mitten Crab (Eriocheir sinensis) Meat Using Electronic Tongue Analysis
}

\author{
Hongbo Liu, ${ }^{1}$ Tao Jiang, ${ }^{1}$ Junren Xue, ${ }^{2}$ \\ Xiubao Chen, ${ }^{1}$ Zhongya Xuan, ${ }^{2}$ and Jian Yang ${ }^{1,2^{*}}$ \\ ${ }^{1}$ Key Laboratory of Fishery Ecological Environment Assessment and Resource Conservation \\ in Middle and Lower Reaches of the Yangtze River, Freshwater Fisheries Research Center, \\ Chinese Academy of Fishery Sciences, Wuxi 214081, China \\ ${ }^{2}$ Wuxi Fisheries College, Nanjing Agricultural University, Wuxi 214081, China
}

(Received May 27, 2021; accepted June 18, 2021)

Keywords: Eriocheir sinensis, taste sensing system, taste-active value, food quality, authentication

An SA402B taste sensing system (electronic tongue) was used to evaluate the meat taste characteristics of three groups of Chinese mitten crab Eriocheir sinensis (H. Milne Edwards, 1853): those from the native culture area in the Yangcheng Lake, Yangcheng Lake-labeled crabs from the market, and those from aquaculture ponds. The electronic tongue data showed that umami was the most predominant taste in the steamed meat, followed by sweetness. The aftertaste of umami was clearly observed in all crab meat samples. Saltiness and bitterness varied widely depending on the geographic origin. There was only a little aftertaste of bitterness and no discernable sourness, astringency, or aftertaste of astringency. Principal component analysis (PCA) exhibited a clear grouping trend among the three crab groups. Linear discriminant analysis revealed that the crab groups can be separated from each other with $100 \%$ accuracy on the basis of the aforementioned measurable taste values. The taste sensing system can accurately profile the taste characteristics of crabs native to Yangcheng Lake, those marketed as Yangcheng Lake-labeled crabs, and those of pond-cultured crabs.

\section{Introduction}

The Chinese mitten crab Eriocheir sinensis (H. Milne Edwards, 1853) is indigenous to China's coastal rivers and estuaries, and its distribution extends to the Yalujiang River, Liaoning Province in the north and to Fujian Province in the south. ${ }^{(1,2)}$ It has spread to northern European countries, as well as areas in western Asia, Canada, and North America. ${ }^{(3)}$ Chinese mitten crabs are catadromous species. ${ }^{(4)}$ They hatch in the sea, migrate into freshwater areas to develop into adults, and then return to the sea to breed. ${ }^{(5,6)}$

Chinese people have been eating crab meat in late fall and early winter for thousands of years, as it is a rich source of protein and minerals. ${ }^{(7)}$ China is the largest aquaculture producer of Chinese mitten crabs worldwide. With the development, application, and promotion of artificial propagation techniques, the crab's annual output reached a record level of 812103 tons, *Corresponding author: e-mail: jiany@ffrc.cn https://doi.org/10.18494/SAM.2021.3444 
and its production generated revenue of $\$ 11.2$ billion in $2016,{ }^{(8)}$ mainly produced by the lacustrine large purse seine and pond culture approaches. Crustaceans of dubious provenance infringe upon the legitimate rights and interests of consumers and undermine market equity. ${ }^{(9)}$ In China, all crabs in markets should be labeled according to their geographic origin. ${ }^{(10)}$ The price of Chinese mitten crabs from different geographic origins differs greatly. For example, Yangcheng Lake, located northwest of Suzhou City in Jiangsu Province, is famous for its high water quality, shallow water and hard bottom, and abundant water grass. This lake provides ideal conditions for Chinese mitten crab growth, giving Yangcheng Lake crab meat a clean and slightly sweet taste. Yangcheng Lake is the most famous aquaculture area of origin for the Chinese mitten crab, and the crabs farmed there remain the best-selling variety of hairy crabs in the country. They are so reputed for their taste that people willingly pay high prices for them. Therefore, unscrupulous vendors have devised a way to earn money by bringing in cheaper crabs raised elsewhere and letting them live in Yangcheng Lake for a few days or weeks, akin to the crabs merely taking a bath in the water. They then sell such crabs as genuine Yangcheng Lake products for higher prices. Authentic Yangcheng Lake crabs are undoubtedly good, but other regions are catching up in terms of quality; therefore, it is necessary to study the characteristics of crabs farmed from other freshwater lakes in China to potentially increase their commercial value.

The flavor and nutritional composition of aquatic food are of high importance. ${ }^{(7,11)}$ Sensory quality has a key influence on consumer preferences and the market price of food. ${ }^{(12)}$ However, it has become evident that relying on a few experts for flavor evaluation is questionable. ${ }^{(13)}$ Because the morphometric characters of E. sinensis populations are very similar, ${ }^{(14)}$ objectivity cannot be guaranteed just by accumulated experience and bodily memory. Therefore, food sensory evaluation methods must be developed and improved.

The electronic tongue system offers satisfactory taste results close to those of a human sensory evaluation. ${ }^{(15)}$ This system adjusts the balance between hydrophobicity and the electric charge of sensor membranes, obtains detectable changes in the membrane potential upon small changes in the amounts of flavoring substances (i.e., the selective response of the sensor to various flavors is realized by adjusting the type and ratio of lipids/polymers in the sensor membrane), and then converts the intensity values of various flavors according to the WeberFechner law. ${ }^{(12,16)}$ This approach has been broadly used to assess the quality of foods owing to its high objectivity, reliability, and reproducibility. ${ }^{(17-19)}$ Therefore, this technique can provide an alternative method for objectively documenting the taste profile characteristics (umami, aftertaste of umami, sweetness, saltiness, bitterness, sourness, astringency, aftertaste of astringency, and aftertaste of bitterness) of meats from E. sinensis crabs originating from Yangcheng Lake, Yangcheng Lake-labeled crabs from the market, and pond-cultured crabs from Xinghua City, approximately $250 \mathrm{~km}$ from Yangcheng Lake. The electronic tongue analysis presented here is expected to provide a theoretical basis for developing a more objective, reliable, and reproducible approach for the taste evaluation of crabs from both origins and for the labeling of cultured E. sinensis crabs. 


\section{Materials and Methods}

\subsection{Crab materials}

Yangcheng E. sinensis crabs (YCES) were collected from the large purse seine culture area in Yangcheng Lake of Suzhou City, and Yangcheng Lake-labeled crabs (SCES) were purchased from the crab market in Wuxi City in October 2019 (Table 1). Pond-cultured E. sinensis crabs (HGES) were obtained from the aquaculture pond area of Jiangsu Honggao Co., Ltd., Xinghua City in October 2019 (Table 1).

\subsection{Electronic tongue measurement}

Studies of taste differences were conducted using the SA402B taste sensing system (INSENT, Tokyo, Japan). A 15-20 g sample of steam-cooked crab meat was placed in a beaker and blended with deionized water (weight ratio 1:10) for homogenization. The homogenate was filtered through Whatman filter paper, cooled on ice, and then centrifuged. We prepared $80 \mathrm{~mL}$ of the meat sample for the measurement of umami, bitterness, astringency, aftertaste of astringency (aftertaste-A), aftertaste of bitterness (aftertaste-B), and aftertaste of umami (aftertaste-U), and $40 \mathrm{~mL}$ of the meat sample supernatant was used for measuring the sweetness. Each sample

Table 1

Biometrics of YCES, SCES, and HGES from Jiangsu Honggao Co., Ltd., Xinghua City, China.

\begin{tabular}{|c|c|c|c|c|c|c|}
\hline Sampling site & $\begin{array}{c}\text { Sample } \\
\text { group code }\end{array}$ & Sample code & $\begin{array}{l}\text { Body length } \\
(\mathrm{mm})\end{array}$ & $\begin{array}{l}\text { Body height } \\
(\mathrm{mm})\end{array}$ & $\begin{array}{l}\text { Body width } \\
(\mathrm{mm})\end{array}$ & $\begin{array}{c}\text { Body weight } \\
(\mathrm{g})\end{array}$ \\
\hline \multirow{8}{*}{ Yangcheng Lake } & \multirow{8}{*}{ YCES } & YCES1 & 62.05 & 33.45 & 72.55 & 156.98 \\
\hline & & YCES2 & 62.35 & 33.70 & 71.25 & 157.95 \\
\hline & & YCES3 & 65.35 & 32.35 & 74.05 & 151.53 \\
\hline & & YCES4 & 55.75 & 29.45 & 58.15 & 92.2 \\
\hline & & YCES5 & 57.15 & 30.36 & 58.25 & 97.5 \\
\hline & & YCES6 & 55.65 & 30.54 & 62.75 & 80.97 \\
\hline & & YCES7 & 58.25 & 32.65 & 64.25 & 113.43 \\
\hline & & YCES8 & 57.65 & 31.55 & 62.05 & 106.23 \\
\hline \multirow{4}{*}{ Market } & \multirow{4}{*}{ SCES } & SCES1 & 61.85 & 32.5 & 68.15 & 157.3 \\
\hline & & SCES2 & 61.35 & 32.35 & 66.4 & 146.75 \\
\hline & & SCES3 & 55.65 & 31.05 & 60.2 & 97.68 \\
\hline & & SCES4 & 57.15 & 31.25 & 68.3 & 103.48 \\
\hline \multirow{6}{*}{$\begin{array}{c}\text { Pond of Jiangsu } \\
\text { Honggao Co., Ltd. }\end{array}$} & \multirow{6}{*}{ HGES } & HGES1 & 64.35 & 33.05 & 72.15 & 171.32 \\
\hline & & HGES2 & 63.8 & 32.4 & 71.25 & 172.58 \\
\hline & & HGES3 & 61.7 & 32.5 & 69.35 & 161.21 \\
\hline & & HGES4 & 61.35 & 34.2 & 68.8 & 124.04 \\
\hline & & HGES5 & 61.75 & 35.7 & 66.3 & 122.67 \\
\hline & & HGES6 & 61.5 & 34.95 & 66.55 & 117.87 \\
\hline
\end{tabular}


measurement under test cup storage conditions was replicated twice, and we also prepared a reference solution $(2.2365 \mathrm{~g} \mathrm{KCl}+0.045 \mathrm{~g}$ tartaric acid), a positive solution $(7.46 \mathrm{~g} \mathrm{KCl}+$ $500 \mathrm{~mL}$ water $+300 \mathrm{~mL}$ ethanol $+0.56 \mathrm{~g} \mathrm{KOH}$, constant volume of $1000 \mathrm{~mL}$ ), and a negative solution (500 mL water, $300 \mathrm{~mL}$ ethanol, $8.3 \mathrm{~mL} \mathrm{HCl}$, constant volume of $1000 \mathrm{~mL}$ ). All cups of the sample and solution were placed in the right position of the electronic tongue and analyzed using a taste sensor program. Note that only liquids can be analyzed using the electronic tongue; the solutions require no pretreatment, except for filtration.

Each sample was measured after the electric potentials of all membranes were stabilized in standard solutions. These standard solutions were prepared by dissolving the respective compound in $1 \mathrm{~L}$ of distilled water, and included a salty solution $(22.37 \mathrm{~g} \mathrm{KCl}$ and $0.045 \mathrm{~g}$ tartaric acid), a sour solution $(2.24 \mathrm{~g} \mathrm{KCl}$ and $0.45 \mathrm{~g}$ tartaric acid dissolved in $1 \mathrm{~L}$ of distilled water), an umami solution [ $2.24 \mathrm{~g} \mathrm{KCl}, 0.045 \mathrm{~g}$ tartaric acid, and $1.87 \mathrm{~g}$ monosodium glutamate (MSG)], a bitter $(+)$ solution ( $2.24 \mathrm{~g} \mathrm{KCl}, 0.045 \mathrm{~g}$ tartaric acid, and $0.04 \mathrm{~g}$ quinine hydrochloride), a bitter (-) solution (2.24 $\mathrm{g} \mathrm{KCl}, 0.045 \mathrm{~g}$ tartaric acid, and $1000 \mathrm{~mL}$ iso- $\alpha$-acid), and an astringent solution ( $2.24 \mathrm{~g} \mathrm{KCl}, 0.045 \mathrm{~g}$ tartaric acid, and $0.05 \mathrm{~g}$ tannic acid). Final sample solutions were prepared by centrifugation for $15 \mathrm{~min}$ at $2200 \times \mathrm{g}$ before analysis.

The sensors CT0, CA0, AAE, C00, and AE1 were used to measure the individual initial tastes of saltiness, acidity, umami, anionic bitterness, and astringency, respectively. ${ }^{(20)}$ The sensor signals of a sample are shown as differences from those of the peptone solution and are converted to measurement intensity values corresponding to the individual tastes using the following formulas: converted value of $\mathrm{CT} 0$ output $(\mathrm{cvCT} 0)=-0.25 \times \mathrm{CT} 0$ signal; converted value of $\mathrm{CA} 0$ output $(\mathrm{cvCA} 0)=0.33 \times \mathrm{CA} 0$ signal; converted value of AAE output $(\mathrm{cvAAE})=$ $-0.16 \times$ AAE signal; converted value of $\mathrm{C} 00$ output $(\mathrm{cvC} 00)=-0.14 \times \mathrm{C} 00$ signal $+0.08 \times \mathrm{CT} 0$ signal; and converted value of AE1 output $(\mathrm{cvAE} 1)=-0.16 \times \mathrm{AE} 1$ signal $+0.16 \times \mathrm{CT} 0$ signal. In general, a converted value of 1.00 corresponds to a $20 \%$ difference from the standard solution in the sensory evaluation. The $\mathrm{C} 00$ and AE1 sensors comprise hydrophobic polymer membranes; thus, they respond to both bitter and astringent compounds as well as some hydrophobic compounds. The cvC00 and cvAE1 outputs indicate that the taste complexities are at low levels (INSENT, manufacturer's manual).

The output value of the reference solution was the control value, i.e., the tasteless point. As the reference solution comprises $\mathrm{KCl}$ and tartaric acid, the tastelessness values of sourness and saltiness are defined as $<-13$ and $<-6$, respectively. The tastelessness of umami, bitterness, astringency, aftertaste-A, aftertaste-B, aftertaste- $\mathrm{U}$, and sweetness are defined as zero or negative values. Measurement values beyond these criteria of tastelessness are used as effective flavor data.

\subsection{Statistical analysis}

The effective flavor data characteristics of steam-cooked crab meat were assessed by radar chart analysis, principal component analysis (PCA), and discriminant function analysis. All calculations were performed using SPSS 20.0 (SPSS Inc., Chicago, IL, USA). 


\section{Results}

\subsection{Taste-active value}

The measured intensity values of various tastes for the YCES, SCES, and HGES groups are presented in Table 2. Because the values of sourness were far lower than -13, and the astringency and aftertaste-A values were far lower than zero, these tastes could be used in priority to other criteria to document the meat profiles. As shown in Table 2 and Fig. 1, the taste values of steamcooked crab meat generally showed the highest intensity in umami (18.7-20.8), followed by sweetness (13.1-15.8), saltiness (10.8-18.0), bitterness (4.8-10.2), and aftertaste-U (3.0-4.7). The aftertaste-B values were usually the lowest (0.1-2.3). Therefore, umami mainly contributed to the taste of crab meat in this study, whereas aftertaste-B was unimportant for taste. Sourness, astringency, and aftertaste-A were not indicated in the taste of crab meats in this study.

\subsection{Taste patterns in different crab groups}

Figure 2 shows the patterns of various crab meat tastes for the YCES, SCES, and HGES groups based on their corresponding measurement intensity values. In comparison with the taste unit values for the YCES crab meat, HGES crab meat presented a richer and less bitter profile,

Table 2

Taste values of umami, aftertaste-U, saltiness, sweetness, bitterness, and aftertaste-B in meat of "market-sized" YCES, SCES, and HGES from Jiangsu Honggao Co., Ltd., Xinghua City, China.

\begin{tabular}{|c|c|c|c|c|c|c|c|c|}
\hline $\begin{array}{l}\text { Sampling } \\
\text { site }\end{array}$ & $\begin{array}{l}\text { Sample } \\
\text { group code }\end{array}$ & Sample code & Umami & Aftertaste-U & Saltiness & Sweetness & Bitterness & Aftertaste-B \\
\hline \multirow{9}{*}{$\begin{array}{l}\text { Yangcheng } \\
\text { Lake }\end{array}$} & \multirow{9}{*}{ YCES } & YCES1 & 19.58 & 3.04 & 10.8 & 13.65 & 5.14 & 0.37 \\
\hline & & YCES2 & 19.02 & 3.12 & 11.3 & 13.13 & 6.31 & 0.56 \\
\hline & & YCES3 & 19.31 & 3.34 & 16.57 & 14.62 & 9.84 & 1.8 \\
\hline & & YCES4 & 18.72 & 3.65 & 17.97 & 14.49 & 10.17 & 2.26 \\
\hline & & YCES5 & 19.94 & 3.17 & 14.29 & 13.51 & 6.94 & 1.02 \\
\hline & & YCES6 & 19.46 & 4.01 & 12.71 & 13.74 & 8.01 & 1.28 \\
\hline & & YCES7 & 20.3 & 3.32 & 14.13 & 13.9 & 7.39 & 1.39 \\
\hline & & YCES8 & 20.37 & 3.22 & 14.58 & 13.21 & 5.52 & 0.53 \\
\hline & & $($ Mean \pm SD $)$ & $19.6 \pm 0.6$ & $3.4 \pm 0.3$ & $14 \pm 2.5$ & $13.8 \pm 0.5$ & $7.4 \pm 1.9$ & $0.7 \pm 0.7$ \\
\hline \multirow{5}{*}{ Market } & \multirow{5}{*}{ SCES } & SCES1 & 19.49 & 4.3 & 13.6 & 14.37 & 7.68 & 0.92 \\
\hline & & SCES2 & 19.66 & 3.99 & 13.55 & 14.22 & 6.72 & 0.72 \\
\hline & & SCES3 & 20.48 & 4.07 & 17.23 & 14.6 & 7.49 & 1.09 \\
\hline & & SCES4 & 20.13 & 4.44 & 13.69 & 15.82 & 9.4 & 1.44 \\
\hline & & $($ Mean \pm SD) & $19.9 \pm 0.5$ & $4.2 \pm 0.2$ & $14.5 \pm 1.8$ & $14.8 \pm 0.7$ & $7.8 \pm 1.1$ & $1.0 \pm 0.3$ \\
\hline \multirow{7}{*}{$\begin{array}{c}\text { Pond of } \\
\text { Jiangsu } \\
\text { Honggao } \\
\text { Co., Ltd. }\end{array}$} & \multirow{7}{*}{ HGES } & HGES1 & 19.8 & 4.05 & 12.81 & 13.84 & 5.03 & 0.15 \\
\hline & & HGES2 & 20.05 & 4.61 & 13.54 & 14.44 & 7.82 & 1 \\
\hline & & HGES3 & 20.25 & 4.25 & 14.48 & 13.58 & 4.99 & 0.19 \\
\hline & & HGES4 & 20.34 & 4.09 & 14.87 & 13.61 & 5.11 & 0.23 \\
\hline & & HGES5 & 20.29 & 4.65 & 14.81 & 14.99 & 7.92 & 1.11 \\
\hline & & HGES6 & 20.76 & 4.52 & 15.9 & 13.73 & 4.88 & 0.11 \\
\hline & & $($ Mean \pm SD) & $20.3 \pm 0.3$ & $4.4 \pm 0.3$ & $14.4 \pm 1.1$ & $14 \pm 0.6$ & $6 \pm 1.5$ & $0.5 \pm 0.5$ \\
\hline
\end{tabular}




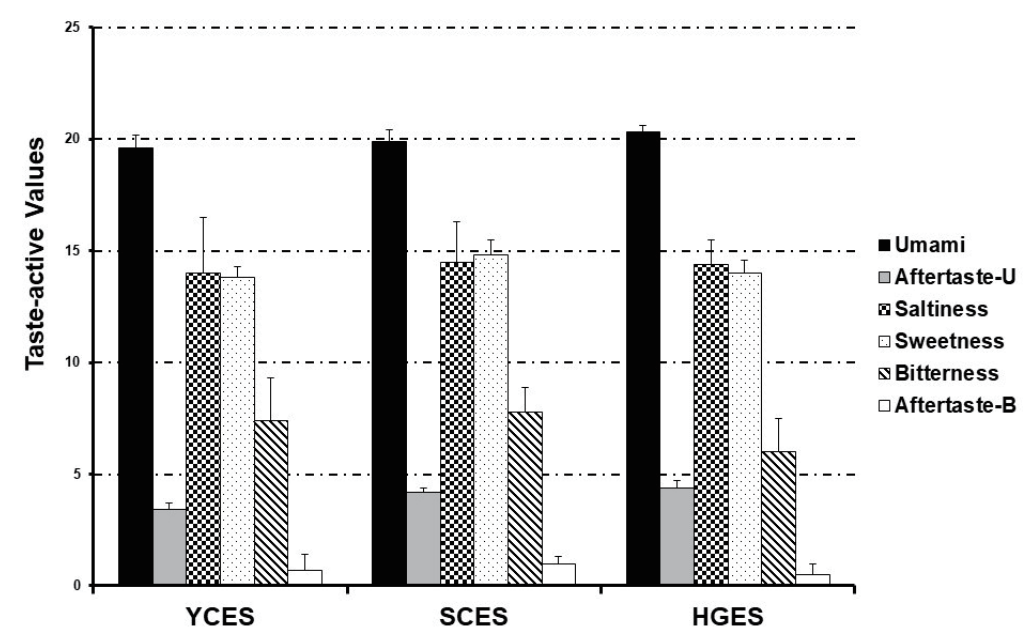

Fig. 1. Taste values of umami, aftertaste-U, saltiness, sweetness, bitterness, and aftertaste-B in steam-cooked meat of YCES, SCES, and HGES from Jiangsu Honggao Co., Ltd., Xinghua City, China.

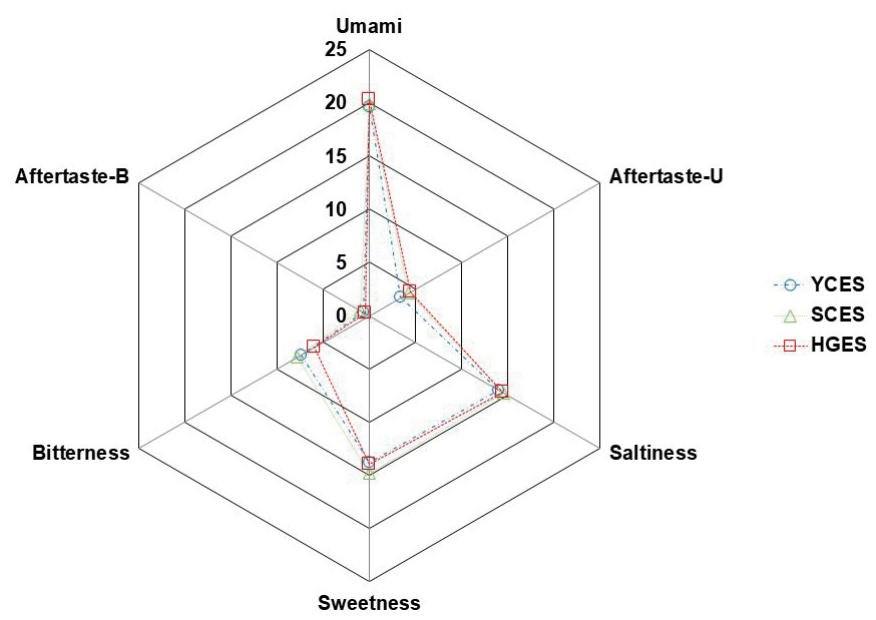

Fig. 2. (Color online) Radar chart of the mean taste values of umami, aftertaste-U, saltiness, sweetness, bitterness, and aftertaste-B in steam-cooked meat of YCES, SCES, and HGES from Jiangsu Honggao Co., Ltd., Xinghua City, China.

whereas SCES crab meat was sweeter and less bitter. Compared with SCES E. sinensis from the market, HGES crabs presented higher aftertaste-U, lower sweetness, and lower bitterness.

\subsection{Multivariate analysis}

Table 3 shows the two-dimensional representation of the PCA for the values of various taste units. The first two components comprised 48.7 and 29.3\% (total 78.1\%) of the variance information accumulated. The main differential features were that principal component 1 (PC1) mainly presented variations in saltiness, sweetness, bitterness, and aftertaste-B, whereas 
Table 3

Loading matrix, eigenvector, and cumulative contribution of principal components of the taste values of umami, aftertaste-U, saltiness, sweetness, bitterness, and aftertaste-B in steam-cooked meat of "market-sized" YCES, SCES, and HGES from Jiangsu Honggao Co., Ltd., Xinghua City, China.

\begin{tabular}{lcccccc}
\hline & \multicolumn{2}{c}{ PC1 } & & \multicolumn{3}{c}{ PC2 } \\
\cline { 2 - 3 } \cline { 6 - 7 } & Loading & Eigenvector & & Loading & Eigenvector \\
\hline Umami & -0.367 & -0.214 & & 0.796 & 0.600 \\
Aftertaste-U & 0.169 & 0.099 & & 0.853 & 0.643 \\
Saltiness & 0.561 & 0.328 & & 0.348 & 0.262 \\
Sweetness & 0.785 & 0.459 & & 0.431 & 0.325 \\
Bitterness & 0.974 & 0.569 & & -0.147 & -0.111 \\
Aftertaste-B & 0.938 & 0.548 & & -0.258 & -0.194 \\
\hline Cumulative contribution (\%) & \multicolumn{3}{c}{48.7} & & \multicolumn{3}{c}{78.1} \\
\hline
\end{tabular}

principal component 2 (PC2) mainly presented variations in umami and aftertaste-U. The PCA score plot (Fig. 3) exhibited a clear grouping trend among the three crab group classifications. In particular, the YCES crab results in Fig. 3 show clusters corresponding to taste values that were clearly different and plotted far away from those of SCES and HGES crabs. The YCES crab results are in quadrants 3 and 4 on the lower side of the figure, indicating that umami and aftertaste-U (i.e., PC2) characteristics mainly explained the separation of the YCES group from the SCES and HGES groups.

Discriminant function analysis of the taste values is shown in Fig. 4. The analyses of umami, aftertaste-U, saltiness, sweetness, bitterness, and aftertaste-B in the steam-cooked meat revealed comprehensive discriminant accuracies of $100 \%$ among the YCES, SCES, and HGES groups of E. sinensis. From the cross-validated rates, data discriminant accuracy was as high as $98.2 \%$. The crabs were classified with successful cross-validated rates of $100 \%$ between the YCES and SCES groups and the YCES and HGES groups and 83.3\% between the SCES and HGES groups.

\section{Discussion}

\subsection{Characteristics of taste profiles}

As a pilot study, this study only focused on the meat of E. sinensis crabs, and did not involve other edible tissues, for example, xiehuang (female crab roe: ovary and digestive glands) and xiegao (crab paste from males: accessory reproductive glands and digestive glands). The results show that the steam-cooked crab meat of all three groups presents the umami taste as the most obvious taste. Umami is one of the most basic tastes, mainly characterized by the representative naturally occurring substance MSG. ${ }^{(21)}$ The T1R1 and T1R3 G-protein-coupled receptor combinations are believed to be constituents of the umami response. ${ }^{(22,23)}$ So far, only Chen and Zhang have investigated the umami taste for E. sinensis. ${ }^{(24)}$ They found that the umami taste in steamed Chinese mitten crab meat was also very intense as the equivalent umami concentration (EUC) was as high as $4.2 \mathrm{~g} \mathrm{MSG} / 100 \mathrm{~g}$, which was eight times higher than the EUC of the snow crab. Moreover, 5'-inosine monophosphate (IMP) and 5'-adenosine monophosphate (AMP) mainly contributed to the strong umami taste.

This study is the first to reveal that sweetness was the second most obvious taste following 


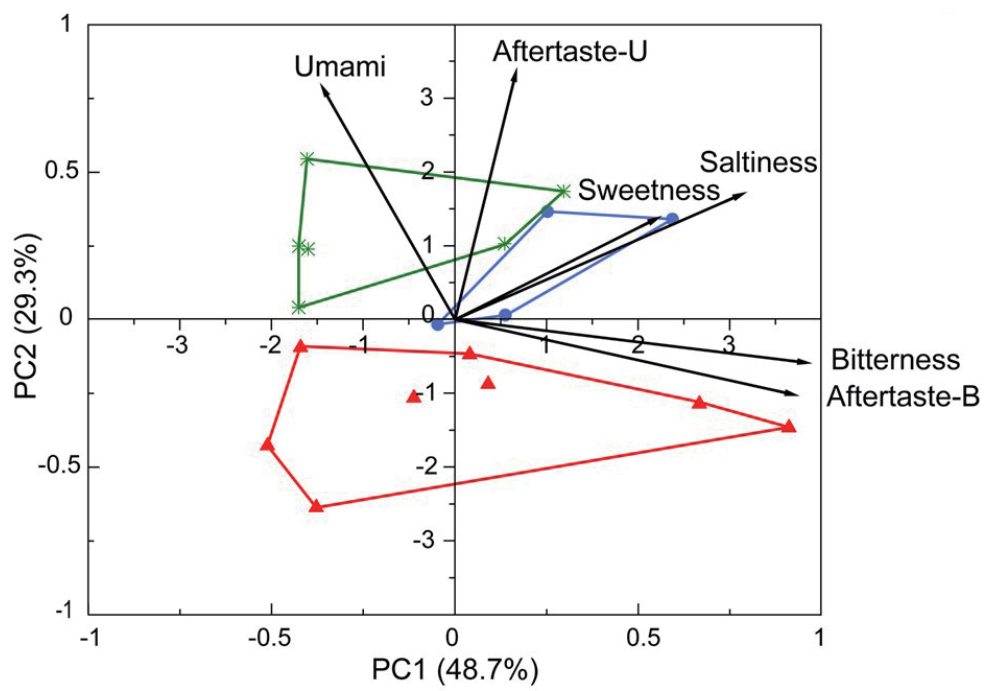

Fig. 3. (Color online) PCA of the taste values of umami, aftertaste-U, saltiness, sweetness, bitterness, and aftertaste-B in steam-cooked meat of YCES (red), SCES (blue), and HGES (green) from Jiangsu Honggao Co., Ltd., Xinghua City, China.

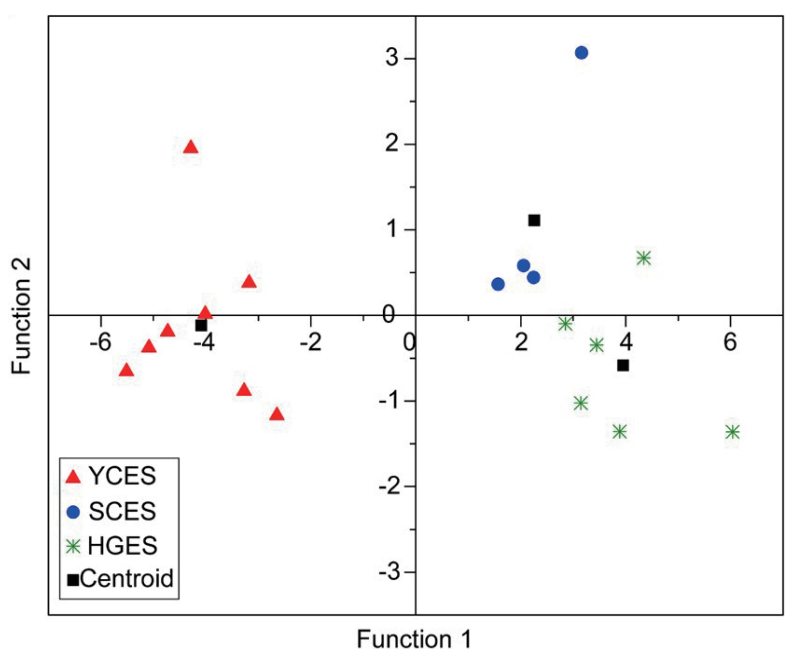

Fig. 4. (Color online) Discriminant function analysis of the taste values of umami, aftertaste-U, saltiness, sweetness, bitterness, and aftertaste-B in steam-cooked meats of YCES (red), SCES (blue), and HGES (green) from Jiangsu Honggao Co., Ltd., Xinghua City, China.

umami, and it documented the taste qualities of crab meat. Glycine and alanine are two of the major free amino acids in crab meat that possibly contribute to the sweet taste. (24) These two types of amino acids can be found not only in crabs but also in fish and squids. ${ }^{(25,26)}$ 
Saltiness is another major taste in crab meat, whose measurement values almost reached the sweetness values in this study. Saltiness is mainly produced by $\mathrm{NaCl}^{(27)}$ and the sodium-specific salt taste is mediated by epithelial sodium channels. ${ }^{(28,29)}$ It is noteworthy that saltiness in fish and crustaceans may be influenced by cations (e.g., $\mathrm{Na}^{+}, \mathrm{K}^{+}$) and anions (e.g., $\mathrm{Cl}^{-}, \mathrm{PO}_{4}{ }^{3-}$ ). ${ }^{(30,31)}$

Notably, the crab meat in this study exhibited a moderate level of bitterness (average 7.1) and little aftertaste-B (average 0.7); however, no sourness, astringency, or aftertaste-A could be measured. In crab meat, the amino acid most highly related to bitterness is arginine. ${ }^{(7)}$ Bitterness is also effective at cutting aftertaste- $U$ and can enhance the balance, complexity, and flavor of food. ${ }^{(12,32)}$ Therefore, bitterness may have the same effect on the flavor of steamed crab meat in this study. The exact taste values in all crab meat samples indicated obvious aftertaste-U (measured values were all $>3$ ). Aftertaste-U is dubbed as the aftertaste of umami or persistent umami taste and plays a key role in the delicious flavor that determines the higher price of food because of its complexity and flavor. ${ }^{(12)}$ Therefore, the above-mentioned obvious aftertaste-U implies that crab meat can provide consumers with a persistent umami taste.

\subsection{Possibility of crab authentication}

Electronic tongue systems measure the consistency of taste, similarly to the human tongue, on the basis of various taste qualities and their quantification rather than the discrimination of individual chemical substances; therefore, these systems are semi-selective or globally selective. ${ }^{(33)}$ This study obtained exact measurements of saltiness, umami, bitterness, aftertaste- $\mathrm{U}$, sweetness, and aftertaste-B intensity values in the crab meat using the SA402B electronic tongue system. All taste value data (Table 2) were in testable scales of the human gustatory system.

Although intergroup and intragroup variations can be found in various taste values, the radar chart and especially the PCA maps and discriminant function analysis reflected the global properties of the meat taste profiles among genuine Yangcheng Lake-cultured crabs, labeled Yangcheng Lake crabs, and pond-cultured crabs from another geographic area. Several multivariate statistical approaches, such as PCA and linear discriminant analysis, have been broadly used for assessment and visualization of the flavor qualities and taste properties of foods. ${ }^{(34-37)}$ It is noteworthy that SCES, HGES, and especially YCES crabs were separated distinctly by PCA, as shown in Fig. 3. This phenomenon indicates that the differentiation of the global properties of measurable tastes among the three groups of E. sinensis crabs is obvious. However, it also raises the question of whether the labeled Yangcheng Lake crabs from the crab market were genuine. Furthermore, the discriminant accuracy rates obtained by linear discriminant analysis of the taste values of umami, aftertaste-U, saltiness, sweetness, bitterness, and aftertaste-B in the steam-cooked meats were 100\% for the SCES, HGES, and YCES groups (Fig. 4). The results show a strong possibility of using differentiation of the global properties of measurable tastes for crab meat assessment (especially the Yangcheng Lake crabs). The differences in taste among SCES, HGES, and YCES crab groups may respond to taste-related biotic/abiotic habitat conditions/heterogeneities during the culture process. Further research is required to identify and explain the corresponding mechanism effectively. 


\section{Conclusions}

The taste profiles of native Yangcheng Lake-cultured crabs, labeled Yangcheng Lake- E. sinensis crabs, and pond-cultured crabs from a different part of Jiangsu Province were comparatively investigated using the SA402B taste sensing system. Umami was the most predominant taste, followed by sweetness. The aftertaste- $U$ of all crab meat samples was obvious. Saltiness and bitterness varied widely among the meats of different origins. A small amount of aftertaste-B was noted, and there was no discernable sourness, astringency, or aftertaste-A. Discriminant accuracy rates by the taste values of umami, aftertaste-U, saltiness, sweetness, bitterness, and aftertaste-B in steam-cooked meats were $100 \%$ for the three crab groups, indicating that the taste sensing system can accurately profile the taste characteristics of "market-sized" Yangcheng Lake, marketed Yangcheng Lake-labeled, and pond-cultured crabs from different culture areas with the possibility of crab authentication.

\section{Acknowledgments}

This work was supported by the National Natural Science Foundation of China (Grant code 31772850), Central Public-interest Scientific Institution Basal Research Fund, CAFS (Grant code 2021XT0704), and the Scientific Research Foundation of Wuxi City for Returned Overseas Chinese (Grant code CZ2018006700).

\section{References}

1 É. Veilleux and Y. de Lafontain: Can. Manuscr. Rep. Fish. Aquat. Sci. 2812 (2007) 1. http://publications.gc.ca/ pub?id $=9.618621 \& \mathrm{sl}=0$

2 J. Garcia-de-Lomas, E. D. Dana, J. López-Santiago, R. González, G. Ceballos, and F. Ortega: Aquat. Invas. 5 (2010) 323. http://doi.org/10.3391/ai.2010.5.3.11

3 L. M. Herborg, S. P. Rushton, A. S. Clare, and M. G. Bentley: Hydrobiologia 503 (2003) 21. http://doi. org/10.1023/B:HYDR.0000008483.63314.3c

4 L. M. Herborg, S. P. Rushton, and A. S. Clare: Biol. Invas. 7 (2005) 959. http://doi.org/10.1007/s10530-0042999-y

5 Q. D. Wang, J. S. Liu, S. Y. Zhang, Y. X. Lian, H. Y. Ding, X. Du, Z. J. Li, and S. De Silva Sena: Ambio 45 (2016) 361. http://doi.org/10.1007/s13280-015-0722-0

6 Z. Cui, Y. Liu, J. Yuan, X. Zhang, T. Ventura, K. Y. Ma, S. Sun, C. Song, D. Zhan, Y. Yang, H. Liu, G. Fan, Q. Cai, J. Du, J. Qin, C. Shi, S. Hao, Q. P. Fitzibbon, G. G. Smith, J. Xiang, T. Y. Chan, M. Hui, C. Bao, F. Li, and K. H. Chu: Nat. Commun. 12 (2021) 2395. https://doi.org/10.1038/s41467-021-22604-3

7 D. W. Chen, M. Zhang, and S. Shrestha: Food Chem. 103 (2007) 1343. http://doi.org/10.1016/j. foodchem.2006.10.047

8 Ministry of Agriculture, China (MOAC): China Fisheries Yearbook 2018 (China Agriculture Publisher, Beijing, 2018).

9 R. J. Luo, T. Jiang, X. B. Chen, C. C. Zheng, H. B. Liu, and J. Yang: Food Chem. 274 (2019) 1. http://doi. org/10.1016/j.foodchem.2018.08.104

10 C. C. Zheng, T. Jiang, R. J. Luo, X. B. Chen, and J. Yang: N. Am. J. Fish. Manag. 41 (2021). http://doi. org/10.1002/nafm.10396

11 M. Yamashita, A. Namikoshi, J. Iguchi, Y. Takashima, T. Yabu, and Y. Yamashita: Fisheries for Global Welfare and Environment, K. Tsukamoto, T. Kawamura, T. Takeuchi, T. D. Beard Jr., and M. J. Kaiser, Eds. (TERRAPUB, Tokyo, 2008) pp. 297-306.

12 K. Toko: Analysis of the Tastes on the Tip of the Tongue. (China Quality Press, China Standard Press, Beijing, 2013) pp. 1-138. 
13 D. Ha, Q. Sun, K. Q. Su, H. Wan, H. B. Li, N. Xu, F. Sun, L. J. Zhuang, N. Hu, and P. Wang: Sens. Actuators, B 207 (2015) 1136. http://doi.org/10.1016/j.snb.2014.09.077

14 W. B. Yang, Y. P. Su, H. B. Liu, and J. Yang: J. Fish. Sci. China 19 (2013) 84. http://doi.org/10.3724/ SP.J.1118.2012.00084

15 Y. Kobayashi, M. Habara, H. Ikezazki, R. G. Chen, Y. Naito, and K. Toko: Sensors 10 (2010) 3411. http://doi. org/10.3390/s100403411]

16 X. Zhang, H. W. Wu, X. K. Yu, H. Y. Luo, Y. Q. Lu, H. J. Yang, X. Li, Z. Y. Li, L. Y. Tang, and Z. Wang: Molecules 23 (2018) 3362. http://doi.org/10.3390/molecules23123362

17 K. Chikuni, M. Oe, K. Sasaki, M. Shibata, I. Nakajima, and K. Ojima: Anim. Sci. J. 81 (2010) 600. http://doi. org/10.1111/j.1740-0929.2010.00773.x

18 D. Liu, S. Li, N. Wang, Y. J. Deng, L. Sha, S. M. Gai, H. Liu, and X. L. Xu: J. Food Sci. 82 (2017) 1076. http:// doi.org/10.1111/1750-3841.13693

19 P. Pattarapon, M. Zhang, B. Bhandari, and Z. X. Gao: J. Food Process. Preserv. 42 (2018) e13418. http://doi. org/10.1111/jfpp.13418

20 H. Kimoto-Nira, R. Aoki, K. Mizumachi, K. Sasaki, H. Naito, T. Sawada, and C. Suzuki: J. Dairy Sci. 95 (2012) 2176. http://doi.org/10.3168/jds.2011-4824

21 S. Yamaguchi: Physiol. Behav. 49 (1991) 833. http://doi.org/10.1016/0031-9384(91)90192-q

22 G. Nelson, J. Chandrashekar, M. A. Hoon, and L. X. Feng: Nature 416 (2002) 199. http://doi.org/10.1038/ nature726

23 G. Q. Zhao, Y. Zhang, M. A. Hoon, J. Chandrashekar, I. Erlenbach, N. J. P. Ryba, and C. S. Zuker: Cell 115 (2003) 255. http://doi.org/10.1016/s0092-8674(03)00844-4

24 D. W. Chen and M. Zhang: Food Chem. 104 (2007) 1200. http://doi.org/10.1016/j.foodchem.2007.01.042

25 S. Fuke and S. Konosu: Physiol. Behav. 49 (1991) 863. http://doi.org/10.1016/0031-9384(91)90195-t

26 D. S. Lee, H. A. Cho, N. Y. Yoon, Y. K. Kim, C. W. Lim, and K. B. Shim: Aqua. Sci. 15 (2012) 99. http://doi. org/10.5657/FAS.2012.0099

27 K. Toko: Sens. Actuators, B 64 (2000) 205. http://doi.org/10.1016/S0925-4005(99)00508-0

28 J. A. DeSimone and V. Lyall: Am. J. Physiol. Gastrointest. Liver Physiol. 291 (2006) G1005. http://doi. org/10.1152/ajpgi.00235.2006

29 S. K. Chay, A. V. Keating, C. James, A. Aliev, S. Haider, and D. Q. M. Craig: RSC Adv. 8 (2018) 3564. http:// doi.org/10.1039/C7RA11015D

30 T. Hayashi, K. Yamaguchi, and S. Konosu: J. Food Sci. 46 (1981) 479. http://doi.org/10.1111/j.1365-2621.1981. tb04890.x

31 Y. Kani, N. Yoshikawa, S. Okada, and A. Hiroki: Food Res. Int. 41 (2008) 371. http://doi.org/10.1016/j. foodres.2008.01.001

32 Y. Komata: Food Rev. Int. 6 (1990) 457. http://doi.org/10.1080/87559129009540887

33 K. Toko and Y. Tahara: Electronic Noses and Tongues in Food Science, M. L. R. Méndez, Ed. (Academic Press, London, San Diego, Cambridge, Oxford, 2016) pp. 161-170.

34 A. Legin, A. Rudnitskaya, Y. Vlasov, C. Di Natale, F. Davide, and A. D'Amico: Sens. Actuators, B 44 (1997) 291. http://doi.org/10.1016/S0925-4005(97)00167-6

35 M. L. R. Méndez: Electronic Noses and Tongues in Food Science (Academic Press, London, San Diego, Cambridge, Oxford, 2016).

36 H. Zhang, G. Zou, X. Liu, Y. Xiao, and W. Wang: Sens. Mater. 31 (2019) 2347. http://doi.org/10.18494/ SAM.2019.2244

37 X. Wu, Y. Tahara, R. Yatabe, and K. Toko: Anal. Sci. 36 (2020) 147. http://doi.org/10.2116/analsci.19R008 
